\title{
Two general methods for the numerical approximation of multidimensional Cauchy principal value integrals
}

\author{
Kai Diethelm*
}

(Received 29 February 2000; revised 7 July 2000)

\begin{abstract}
The numerical approximation of integrals containing strongly singular integrals, in particular Cauchy principal value integrals, is a major issue connected, e.g., to the boundary integral approach for many types of partial differential equations. Whereas the one-dimensional

*Institut für Angewandte Mathematik, Technische Universität Braunschweig, Pockelsstraße 14, D-38106 Braunschweig, Germany. mailto:k.diethelm@tu-bs.de

${ }^{0}$ See http: //anziamj . austms .org.au/V42/E016 for this article and ancillary services, (C) Austral. Mathematical Soc. 2000. Published 21 July 2000, amended 24 July 2000.
\end{abstract}


problem has been addressed very intensively in recent years, much less attention has been paid to multidimensional problems. In the present paper, we investigate two possible approaches to this problem, corresponding to generalizations of two approaches known in the 1-D case. In principle, both methods can be applied to integration domains of arbitrary shape, although we find that certain combinations of algorithms and domains are more useful than others. In particular, we discuss error estimates and show that the methods are highly competitive. Moreover, in contrast to most of the previously discussed methods, the approaches are very efficient when integrals have to be calculated for various locations of the singularity.

\section{Contents}

1 Introduction

2 The Interpolation Operator

5.1 Integrals in more than two dimensions . . . . . . . . . . . E18

5.2 Integrals on domains of general shape . . . . . . . . . . . E19 
5.3 Integrals with singularities of higher order . . . . . . . . . E21

5.4 Surface integrals . . . . . . . . . . . . . . E23

References

E24

\section{Introduction}

The topic of this paper is the numerical approximation of Cauchy principal value integrals in more than one dimension. Following [14, Ch. 8], we concentrate our attention on integrals of the form

$$
I[f](t):=\int_{B} \frac{f(x)\langle v, x-t\rangle}{\|x-t\|^{d+1}} d x:=\lim _{\epsilon \rightarrow 0+} \int_{B \backslash B_{\epsilon}(t)} \frac{f(x)\langle v, x-t\rangle}{\|x-t\|^{d+1}} d x .
$$

Here $B$ is a $d$-dimensional domain contained in $\mathbb{R}^{k}$ where $k=d$ or $k=d+1$, and $t \in B$. Moreover, $v \in \mathbb{R}^{k}$ is an arbitrary given vector, $\langle\cdot, \cdot\rangle$ is the standard inner product in $\mathbb{R}^{k}$, the norm is the usual Euclidean norm, and $B_{\epsilon}(t):=\{x \in B:\|x-t\|<\epsilon\}$. Integrals of this form play an important role when partial differential equations are recast into boundary integral equations in order to be solved, e.g., by the boundary element method (BEM) [10, 11, $14,18,19,20]$. Note that, since the order of the singularity is the same as the dimension of the domain of integration, the integral will usually not exist in the classical sense. However, it is known that under rather weak assumptions on the integrand function $f$, the limit mentioned in (1) will exist. 
For our purposes, the following very simple criterion [14, Lemma 8.1.11] will be sufficient.

Lemma 1 Assume that the function $f$ satisfies a Hölder condition of order $\nu>0$. Then, $I[f](t)$ exists for every $t$ in the interior of $B$.

A vast number of publications dealing with this problem in $\mathbb{R}^{1}$ has appeared in recent years, but for the multidimensional problem only a few results are known, mainly discussing the two-dimensional case [3, 5, 16, 17, 20]. Our methods can be applied to problems in any number of dimensions, and the domains of integration can be of (almost) completely arbitrary shape.

We present two different methods that can be applied to evaluate the integral (1) numerically. Our investigations focus on error estimates, but numerical stability problems will also be addressed. Moreover, the methods are derived in such a way that an efficient calculation of $I[f](t)$ is possible even in the case that approximations are sought for many different values of $t$. The key to this efficiency is simply to choose (most of) the nodes of the quadrature formulas independent of the location of $t$. Note that frequently this issue is not taken into consideration [3, 20].

In classical applications, the BEM is normally used for linear equations only. In this case, the range of integration $B$ of the singular integrals is usually the boundary of the domain where the solution of the partial differential equation is sought. Typically, in such a situation the set $B$ itself does not 
have a boundary (e.g., if the PDE is to be solved on a ball, $B$ would be a sphere). In recent work some authors have tried to extend the BEM idea to nonlinear problems [1, pp. 255ff.]. Doing so, it turns out that Cauchy singular integrals arise whose domain of integration is the entire area where the PDE is given, which has a boundary. Some of the classical methods used for the numerical approximation of (1) perform very badly if $t$ is close to the boundary of $B$. We shall see this in $\S 3$. Therefore, we derive alternative methods avoiding these problems.

In all cases, the quadrature formulas will be based on the combination of the Cauchy integral operator and a spline interpolation operator. We also interpret this spline interpolation operator in the spirit of a finite-element technique. The spline approach is known to be both conceptually simple and highly efficient in numerical work. For the problem of one-dimensional integrals with strong singularities, this has been discussed in [9].

The remainder of this paper is organized as follows. In the next section we introduce the spline interpolation operator that our methods will be based upon. Sections 3 and 4 contain the descriptions and investigations of our two types of quadrature formulas, and in $\S 5$ we state some general remarks and discuss possible generalizations. 


\section{The Interpolation Operator}

As mentioned in the introduction, all our quadrature formulas are based on the same interpolation operator, which is used in different ways. We now start by describing this operator. For the sake of simplicity, we momentarily restrict our attention to two-dimensional regions $B \subset \mathbb{R}^{2}$, and moreover we assume that the boundary of $B$ is a polygon that nowhere touches itself. The generalization to domains of higher dimensions and other shapes will be discussed later.

Given a such a domain $B$, we follow the classical finite-element idea and introduce a triangulation, i.e. we divide $B$ into a number of triangles $T_{j}$, $j=1,2, \ldots, N_{T}$, such that the following properties hold:

1. $\bigcup_{j=1}^{N_{T}} T_{j}=B$;

2. for $j_{1} \neq j_{2}$, the intersection $T_{j_{1}} \cap T_{j_{2}}$ is either empty or consists of one point or one complete edge of both triangles;

3. all interior angles of all triangles are bounded from below by a constant $\theta>0$

4. $\max _{j} \operatorname{diam} T_{j} \leq h$;

where $\operatorname{diam} T:=\sup _{x, y \in T}\|x-y\|$ is the diameter of $T$. Then we say that $B_{h}:=\left\{T_{j}: j=1,2, \ldots, N_{T}\right\}$ is an $h$-triangulation of $B$. 
On each triangle $T_{j}$, we interpolate the function $f$ with a polynomial of degree $m \geq 1$ in such a way that the resulting interpolating function is continuous on $B$. The way we choose to proceed here is the classical way of achieving this goal. Denoting the corners of $T_{j}$ by $T_{j}^{1}, T_{j}^{2}$, and $T_{j}^{3}$, we take the points

$$
\frac{1}{m}\left(\mu_{1} T_{j}^{1}+\mu_{2} T_{j}^{2}+\mu_{3} T_{j}^{3}\right), \quad \mu_{1}, \mu_{2}, \mu_{3} \in\{0,1, \ldots, m\}, \quad \mu_{1}+\mu_{2}+\mu_{3}=m
$$

as interpolation nodes. It is well known that this choice of nodes leads to a unique solution of the interpolation problem, and moreover the resulting piecewise polynomial is continuous on $B$. The function interpolating $f$ defined in this way will be called $\pi_{h}[f]$.

For the approximation properties of the piecewise polynomial chosen in this way, we have the following error estimate [2].

Lemma 2 Let $f \in C^{\mu+1}(B)$ where $\mu \leq m$, and let $\pi_{h}[f]$ be constructed according to the description above, then

$$
\left\|f-\pi_{h}[f]\right\|_{\infty}=\mathcal{O}\left(h^{\mu+1}\right) \quad \text { and } \quad\left\|\nabla\left(f-\pi_{h}[f]\right)\right\|_{\infty}=\mathcal{O}\left(h^{\mu}\right)
$$

where the norms are Chebyshev norms taken over $B$, and $\nabla$ is the usual gradient operator.

Obviously we may find such a triangulation for every domain $B$ bounded by a polygon if $h$ is sufficiently small. We have thus found a good approximation for $f$ whose gradient simultaneously is a good approximation of the 
gradient of $f$. This will be the key to the error bounds for the quadrature methods. Similar observations have been made for the one-dimensional quadrature problem [9].

Before we come to our quadrature formulas, we state one more property of the function $\pi_{h}[f]$ that we use later on.

Lemma 3 Under the assumptions mentioned above, for any given triangulation $B_{h}, \pi_{h}[f]$ fulfils a Hölder condition of order one on $B$.

Proof: The proof is essentially the same as the proof for the corresponding one-dimensional problem. First note that $\pi_{h}[f]$ is a polynomial on every triangle $T_{j}$. Thus it is differentiable there, and in particular it fulfils a Hölder condition of order one with Hölder constant $L_{j}$. Moreover, because of the continuity of $\pi_{h}[f]$ on $B$, the Hölder property is not lost as we move from one triangle to an adjacent triangle. Since we only have finitely many triangles, $L:=\max _{j} L_{j}$ exists, and therefore $\pi_{h}[f]$ fulfils a global Hölder condition on $B$ with Hölder constant $L$.

\section{A Product Quadrature Method}

The product quadrature method is probably the simplest method: we simply replace $f$ by $\pi_{h}[f]$ in (1). Thus our product quadrature formula $Q_{h}$ is defined 
according to

$$
Q_{h}[f](t):=I\left[\pi_{h}[f]\right](t) .
$$

It is an obvious consequence of Lemmas 1 and 3 that $Q_{h}[f](t)$ exists for every $t$ in the interior of $B$. Of course, for a practical implementation it is not sufficient to know only that the required approximation exists, one also needs a concrete formula for $Q_{h}[f]$. To write such a formula is possible in view of the structure described in (3). For example, one can use the classical method of the subtraction of the singularity:

$$
Q_{h}[f](t)=I\left[\pi_{h}[f]-\pi_{h}[f](t)\right](t)+\pi_{h}[f](t) I[1](t) .
$$

Computer algebra systems like Mathematica handle the second integral; for the first integral we notice that the singularity has disappeared. We may perform a polynomial division to rewrite this integral as an integral over a polynomial multiplied by a weakly singular function. Both the polynomial division and the actual integration can be then also delegated to a computer algebra system. The resulting formulas are rather lengthy, therefore we refrain from explicitly mentioning them here.

One of the particularly attractive features of this method is that the location of the quadrature nodes (these being identical to the interpolation nodes) is completely independent of $t$. This means that if we need to approximate $I[f](t)$ for a range of different values of $t$, we only need to perform the function evaluations of $f$ once. These values may then be re-used when $t$ is changed. Additional function evaluations are not necessary. Many of the previously discussed methods $[3,17,20]$ do not share this property. A method 
that does implement the re-use of functional values has been proposed recently in [13]. (I thank an anonymous referee for drawing my attention to this as yet unpublished reference.)

For this method, we derive the following error estimate.

Theorem 4 Let $m, B, B_{h}$ and $\pi_{h}$ be as stated in $\S 2$. Moreover, let $f \in$ $C^{\mu+1}(B)$ where $\mu \leq m$, and let $B^{*}$ be a closed subset of the interior of $B$, then

$$
I[f](t)-Q_{h}[f](t)=\mathcal{O}\left(h^{\mu+1} \ln h^{-1}\right)
$$

holds uniformly for all $t \in B^{*}$.

Note that, under our assumptions on the function $f$, it is not possible to construct a quadrature formula with a smaller uniform error bound. This follows since the ideas used for the corresponding result in the one-dimensional setting $[6, \S 3]$ can be carried over to the multidimensional problem without any major modifications. In other words, the error term of the quadrature formula $Q_{h}$ is of optimal order.

Proof: By assumption, there exists a $\delta>0$, independent of $t$, such that for all $t \in B^{*}$ we have $\left\{x \in \mathbb{R}^{2}:\|x-t\| \leq \delta\right\} \subset B$. Since we are interested in an asymptotic estimate as $h \rightarrow 0$, we assume in the present proof that $h<\delta$. 
Applying the definition of $Q_{h}$ we obtain

$$
I[f](t)-Q_{h}[f](t)=\int_{B \backslash B_{h}(t)} \frac{r_{h}(x)\langle v, x-t\rangle}{\|x-t\|^{3}} d x+\int_{B_{h}(t)} \frac{r_{h}(x)\langle v, x-t\rangle}{\|x-t\|^{3}} d x,
$$

where $r_{h}:=f-\pi_{h}[f]$, and $B_{h}(t):=\left\{x \in \mathbb{R}^{2}:\|x-t\|<h\right\} \subseteq B$. We bound the two integrals in the following way. For the first integral

$$
\left|\int_{B \backslash B_{h}(t)} \frac{r_{h}(x)\langle v, x-t\rangle}{\|x-t\|^{3}} d x\right| \leq\left\|r_{h}\right\|_{\infty} \int_{C \backslash B_{h}(t)} \frac{|\langle v, x-t\rangle|}{\|x-t\|^{3}} d x
$$

where $C$ is the smallest circle with centre at $t$ such that $B \subseteq C$. Because of the boundedness of $B$, we see that the radius of $C$ is bounded from above by an absolute constant $\rho$ independent of $t$. Introducing polar coordinates and applying Lemma 2, we derive

$$
\begin{aligned}
\left|\int_{B \backslash B_{h}(t)} \frac{r_{h}(x)\langle v, x-t\rangle}{\|x-t\|^{3}} d x\right| & \leq \mathcal{O}\left(h^{\mu+1}\right) \int_{0}^{2 \pi} \int_{h}^{\rho} \frac{\left|v_{1} r \cos \theta+v_{2} r \sin \theta\right|}{r^{2}} d r d \theta \\
& \leq \mathcal{O}\left(h^{\mu+1}\right) \int_{0}^{2 \pi}\left|v_{1} \cos \theta+v_{2} \sin \theta\right| d \theta \int_{h}^{\rho} \frac{1}{r} d r \\
& =\mathcal{O}\left(h^{\mu+1} \ln h^{-1}\right)
\end{aligned}
$$

uniformly for $t \in B^{*}$. To bound the remaining integral in (4), first note that an explicit calculation reveals

$$
\int_{B_{h}(t)} \frac{\langle v, x-t\rangle}{\|x-t\|^{3}} d x=0 .
$$


Using this identity and Taylor's theorem, we rewrite the integral in question according to

$$
\begin{aligned}
\int_{B_{h}(t)} \frac{r_{h}(x)\langle v, x-t\rangle}{\|x-t\|^{3}} d x & =\int_{B_{h}(t)} \frac{\left(r_{h}(x)-r_{h}(t)\right)\langle v, x-t\rangle}{\|x-t\|^{3}} d x \\
& =\int_{B_{h}(t)} \frac{\langle v, x-t\rangle \nabla r_{h}(\xi)}{\|x-t\|^{2}} d x
\end{aligned}
$$

where $\xi \in B$. Now use Lemma 2 and introduce polar coordinates once again to obtain

$$
\begin{aligned}
\left|\int_{B_{h}(t)} \frac{r_{h}(x)\langle v, x-t\rangle}{\|x-t\|^{3}} d x\right| & \leq \mathcal{O}\left(h^{\mu}\right) \int_{B_{h}(t)} \frac{|\langle v, x-t\rangle|}{\|x-t\|^{2}} d x \\
& \leq \mathcal{O}\left(h^{\mu}\right) \int_{0}^{2 \pi} \int_{0}^{h}\left|v_{1} \cos \theta+v_{2} \sin \theta\right| d r d \theta \\
& =\mathcal{O}\left(h^{\mu+1}\right) .
\end{aligned}
$$

This bound also holds uniformly for all $t \in B^{*}$. Combining the bounds, we find the desired result.

Note that the statement of Theorem 4 implies convergence in a pointwise sense for every $t$ in the interior of $B$. However, uniform convergence cannot be achieved by this method in general. This is, in fact, the major disadvantage of the product quadrature approach. The proof of this claim follows from the next result. 
Theorem 5 Let $B, B_{h}$ and $\pi_{h}$ be as stated in $\S 2$. Moreover, let $f \in C^{1}(B)$ and assume that there exists a triangle $T_{j} \in B_{h}$ such that the restriction of $f$ onto $T_{j}$ is not a polynomial, then

$$
\sup _{t \in B}\left|I[f](t)-Q_{h}[f](t)\right|=\infty .
$$

Proof: Since $f$ is not a polynomial on $T_{j}$, there exists a point $t^{*}$ at the boundary of $B$ and at the boundary of $T_{j}$ such that $f\left(t^{*}\right) \neq \pi_{h}[f]\left(t^{*}\right)$. Now, defining $r_{h}:=f-\pi_{h}[f]$, we see that

$$
\begin{aligned}
\sup _{t \in B}\left|I[f](t)-I\left[\pi_{h}[f]\right](t)\right| & \geq \limsup _{t \rightarrow t^{*}}\left|I\left[r_{h}\right](t)\right| \\
& =\limsup _{t \rightarrow t^{*}}\left|I\left[r_{h}-r_{h}\left(t^{*}\right)\right](t)+r_{h}\left(t^{*}\right) I[1](t)\right| \\
& \geq \limsup _{t \rightarrow t^{*}}\left(\left|r_{h}\left(t^{*}\right) I[1](t)\right|-\left|I\left[r_{h}-r_{h}\left(t^{*}\right)\right](t)\right|\right) .
\end{aligned}
$$

Looking at these terms independently, we derive that, since the function $r_{h}-r_{h}\left(t^{*}\right)$ vanishes at $t^{*}$, the expression $I\left[r_{h}-r_{h}\left(t^{*}\right)\right](t)$ remains bounded as $t \rightarrow t^{*}$. Recalling that $|I[1](t)|$ diverges to infinity as $t$ approaches the boundary of $B$ and that $r_{h}\left(t^{*}\right) \neq 0$, we obtain that the upper limit is indeed infinite.

One of the key points in this argument is that $B$ does have a boundary. A statement like that of Theorem 5 need not hold, e.g., in situations where $B$ is the surface of a cube or a sphere in $\mathbb{R}^{3}$ that we will address below. 


\section{A Quadrature Formula of the Third Kind}

In the one-dimensional case, essentially two ideas have been proposed to overcome the difficulty of the non-uniform convergence of the product quadrature rules: modified methods $[12, \S 3.2 .1]$; and formulas of the third kind, cf. [7, 15] and the references cited therein. Although the modified methods are very attractive because of their simplicity and their nice theoretical convergence behaviour [8] when the integrand function is smooth, they show severe numerical instabilities when applied practically and may even diverge if the integrand is not smooth [4]. Therefore, we concentrate on formulas of the third kind. They are known [7] not to have these problems.

A quadrature formula of the third kind, $Q_{h}^{(3)}$, for the Cauchy principal value integral (1) is constructed as follows. We start by rewriting the integral according to

$$
I[f](t)=\int_{B}(f(x)-f(t)) \frac{\langle v, x-t\rangle}{\|x-t\|^{3}} d x+f(t) \int_{B} \frac{\langle v, x-t\rangle}{\|x-t\|^{3}} d x,
$$

then we replace the function $f$ in its two occurrences in the first integral by the approximation $\pi_{h}[f]$. The factor $f(t)$ in front of the second integral remains unchanged. Thus, the quadrature formula is defined by

$$
Q_{h}^{(3)}[f](t):=\int_{B}\left(\pi_{h}[f](x)-\pi_{h}[f](t)\right) \frac{\langle v, x-t\rangle}{\|x-t\|^{3}} d x+f(t) \int_{B} \frac{\langle v, x-t\rangle}{\|x-t\|^{3}} d x .
$$

As far as the practical evaluation of this formula is concerned, much the same 
points apply as already mentioned in the previous section when discussing the product quadrature approach.

For these quadrature formulas, we prove a uniform error bound that is of the same order of magnitude as the bound provided for the product rules in Theorem 4, and thus is also of optimal order.

Theorem 6 Let $m, B$ and $\pi_{h}$ be as stated in $\S 2$. Moreover, let $f \in C^{\mu+1}(B)$ where $\mu \leq m$, then

$$
I[f](t)-Q_{h}^{(3)}[f](t)=\mathcal{O}\left(h^{\mu+1} \ln h^{-1}\right)
$$

holds uniformly for all $t \in B$.

Proof: By construction,

$$
I[f](t)-Q_{h}^{(3)}[f](t)=\int_{B}\left\{f(x)-f(t)-\left(\pi_{h}[f](x)-\pi_{h}[f](t)\right)\right\} \frac{\langle v, x-t\rangle}{\|x-t\|^{3}} d x .
$$

As in the proof of Theorem 4, we split the integral into one part covering the integration region $B_{h}(t)=\{x \in B:\|x-t\| \leq h\}$, and one part covering the remainder of $B$. For the first of these integrals, we derive

$$
\left|\int_{B_{h}(t)}\left\{f(x)-f(t)-\left(\pi_{h}[f](x)-\pi_{h}[f](t)\right)\right\} \frac{\langle v, x-t\rangle}{\|x-t\|^{3}} d x\right|
$$




$$
\begin{aligned}
& \leq \int_{B_{h}(t)}\left|\frac{f(x)-f(t)-\left(\pi_{h}[f](x)-\pi_{h}[f](t)\right)}{\|x-t\|} \frac{\langle v, x-t\rangle}{\|x-t\|^{2}}\right| d x \\
& \leq\left\|\nabla\left(f-\pi_{h}[f]\right)\right\| \int_{B_{h}(t)} \frac{|\langle v, x-t\rangle|}{\|x-t\|^{2}} d x
\end{aligned}
$$

by an application of the multidimensional version of Taylor's theorem. Lemma 2 yields that the first factor here is $\mathcal{O}\left(h^{\mu}\right)$ uniformly for all $t$, and an explicit calculation for the second factor reveals

$$
\int_{B_{h}(t)} \frac{|\langle v, x-t\rangle|}{\|x-t\|^{2}} d x \leq \int_{0}^{2 \pi} \int_{0}^{h}\left|v_{1} \cos \theta+v_{2} \sin \theta\right| d r d \theta \leq \mathcal{O}(h),
$$

also uniformly for all $t$. Thus, we have the uniform bound

$$
\left|\int_{B_{h}(t)}\left(f(x)-f(t)-\left(\pi_{h}[f](x)-\pi_{h}[f](t)\right)\right) \frac{\langle v, x-t\rangle}{\|x-t\|^{3}} d x\right|=\mathcal{O}\left(h^{\mu+1}\right) .
$$

For the remaining integral, we proceed as follows. We write

$$
\begin{aligned}
& \left|\int_{B \backslash B_{h}(t)}\left\{f(x)-f(t)-\left(\pi_{h}[f](x)-\pi_{h}[f](t)\right)\right\} \frac{\langle v, x-t\rangle}{\|x-t\|^{3}} d x\right| \\
& \quad \leq 2\left\|f-\pi_{h}[f]\right\| \int_{B \backslash B_{h}(t)} \frac{|\langle v, x-t\rangle|}{\|x-t\|^{3}} d x \\
& \quad \leq \mathcal{O}\left(h^{\mu+1}\right) \int_{0}^{2 \pi} \int_{h}^{\rho}\left|v_{1} \cos \theta+v_{2} \sin \theta\right| \frac{1}{r} d r d \theta
\end{aligned}
$$


where we have again applied Lemma 2 and where $\rho$ is as in the proof of Theorem 4. The last integral behaves as $\mathcal{O}\left(\ln h^{-1}\right)$ uniformly for all $t$, and so we obtain the uniform bound

$\left|\int_{B \backslash B_{h}(t)}\left\{f(x)-f(t)-\left(\pi_{h}[f](x)-\pi_{h}[f](t)\right)\right\} \frac{\langle v, x-t\rangle}{\|x-t\|^{3}} d x\right|=\mathcal{O}\left(h^{\mu+1} \ln h^{-1}\right)$.

Combining these bounds, we obtain the desired result.

Note that we have a small price to pay for the improved behaviour of the method of the third kind as compared to the product method. When calculating $Q_{h}^{(3)}[f](t)$, we have to evaluate $f$ not only at the nodes of $\pi_{h}$, but also at the additional point $t$. Since, in most cases, the number of points to be used for $t$ is significantly smaller than the number of nodes of the interpolation operator, this additional labour is usually likely to be negligible.

We also remark that the two quadrature formulas under consideration are related to each other by

$$
Q_{h}^{(3)}[f](t)=Q_{h}[f](t)+\left(f(t)-\pi_{h}[f](t)\right) I[1](t) .
$$

Therefore, their remainder terms $R_{h}$ and $R_{h}^{(3)}$ are related according to

$$
R_{h}^{(3)}[f](t)=R_{h}[f](t)-\left(f(t)-\pi_{h}[f](t)\right) I[1](t) .
$$

It is an easy consequence of this observation that $Q_{h}^{(3)}[f](t)=Q_{h}[f](t)$ whenever $t$ is one of the nodes of the interpolation operator $\pi_{h}$. Moreover, by 
combining eq. (8) with Theorem 6 and Lemma 2, we obtain an alternative proof of Theorem 4. Additionally, we can provide a second proof of Theorem 5 by means of eq. (8), Theorem 6 , and some elementary properties of the Cauchy integral operator $I$.

\section{$5 \quad$ Generalizations and Remarks}

Having discussed the algorithms for two-dimensional polygonal regions embedded in $\mathbb{R}^{2}$, we now look at various extensions. In particular, we look at integrals in higher dimensions and at integrals over non-polygonal regions. Then we mention integrals where the weight functions involved possess singularities of even higher order. Finally, we briefly discuss how to carry over our methods to integrals over two-dimensional manifolds embedded in $\mathbb{R}^{3}$ which is very important in the classical boundary element method.

Although all these generalizations and extensions will be treated separately, it is easily seen that two or more of them can be combined without any problems.

\subsection{Integrals in more than two dimensions}

The first generalization we present is the one to an arbitrary number of dimensions. This means that we now approximate integrals of the form (1) 
where $B$ is a $d$-dimensional domain in $\mathbb{R}^{d}$, and we drop the restriction that $d=2$. In this case, the results of [2] still allow us to split the domain into subdomains having the shape of $d$-dimensional simplices. Then we construct the corresponding interpolation operators mapping the continuous functions onto continuous piecewise polynomials in $d$ variables. The error estimates of $\S 2$ remain valid if we take into consideration that the condition on the smallest angle of the triangles mentioned in $\S 2$ must be replaced by a condition relating the inscribed spheres of the sub-simplices to the circumscribed spheres [2, p. 179].

As a consequence of these observations, we find that Theorems 4, 5, and 6 remain true also in higher-dimensional spaces. Of course, we must not forget that even though the error estimates remain independent of the dimension $d$, the amount of computational work involved depends on $d$ since the number of interpolation nodes is $\mathcal{O}\left(h^{-d}\right)$. Therefore, the methods are likely to be useful for small and moderately large values of $d$, but for very large $d$ the complexity may be prohibitive for practical applications.

\subsection{Integrals on domains of general shape}

Next we discuss the situation where the boundary of the domain of integration $B$ is not a polygon. To cover the cases that are interesting for the usual applications, we assume that the boundary of $B$, which we shall denote by $\partial B$, is a piecewise $C^{1}$ curve that nowhere touches itself. In this case, we 
proceed as follows.

Given the meshsize $h$, we approximate the set $B$ by a polygon $P_{h}$ with boundary $\partial P_{h}$ (that does not touch itself) in such a way that the following conditions are satisfied:

1. all the points of $P_{h}$ are contained in $B \cup \partial B$;

2. $K_{h}:=\partial B \cap \partial P_{h} \neq \emptyset$;

3. $\sup _{x \notin P_{h}} \min _{y \in P_{h}}\|x-y\| \leq h$.

The essence of this construction is as follows. The polygon $P_{h}$ is inscribed into the arbitrarily shaped domain $B$ such that all points not covered by $P_{h}$ are at most $h$ length units apart from a point in $P_{h}$.

We again construct our two quadrature formulas according to the methods described above. However, we need to say some more words about the definition of the interpolation operator $\pi_{h}$. On the interior of the auxiliary polygon $P_{h}$, we define $\pi_{h}$ in the way mentioned above. Then we note that the remaining part of $B$ can be decomposed into a finite number of disjoint regions. Each of these regions shares at least one edge with the polygon $P_{h}$, and therefore also with one of the sub-triangles of $P_{h}$, say $T^{*}$. On $T^{*}$ we know that $\pi_{h}[f](x)=q(x)$ with some polynomial $q$. For $x$ in the region in question adjacent to $T^{*}$ we simply set $\pi_{h}[f](x)=q(x)$ as well. In this way we extend the definition of the interpolation operator to all of $B$. The three 
conditions stated above ensure that the error bounds of $\S 2$ remain valid, and therefore the quadrature error bounds of $\S \S 3$ and 4 also remain true.

\subsection{Integrals with singularities of higher order}

Up to this point, we have only discussed the case that the order of the singularity coincides with the dimension of the domain of integration. This is the classical problem that arises in most of the traditional BEM approaches. However, in recent years more attention has been focused onto formulations involving singularities of higher order $[14, \S 8.3]$. We thus consider integrals of the form

$$
I_{p}[f](t):=\int_{B} \frac{f(x)\langle v, x-t\rangle}{\|x-t\|^{p}} d x
$$

or

$$
I_{p}^{*}[f](t):=\int_{B} \frac{f(x)\langle v, x-t\rangle\langle w, x-t\rangle}{\|x-t\|^{p}} d x
$$

where $p \geq \operatorname{dim} B+1$. The latter form arises, e.g., when two normal derivative operators are applied in the derivation of a boundary integral equation. Up to a certain extent, our quadrature formulas cope with these integrals too.

The main problem here is more of analytic nature: We need to restrict $p$ in order to make sure that an analogue of Lemma 1 holds. We need to have such a Lemma because otherwise it is not assured that our quadrature formulas exist. The precise formulation goes along the following lines. 
Lemma 7 Assume that the function $f$ satisfies a Hölder condition of order $\nu$, then $I_{p}[f](t)$ exists for every $t$ in the interior of $B$ if $p$ satisfies the restriction

$$
p<1+\nu+\operatorname{dim} B
$$

The proof is very similar to the proof of Lemma 1 (cf. [14, Lemma 8.1.11]) and is therefore omitted.

We have seen in Lemma 3 that in the case considered here, $\pi_{h}[f]$ satisfies a Hölder condition of order 1 . Thus, our quadrature formulas exist whenever $p<2+\operatorname{dim} B$. Under this assumption, we can carry over the proofs of the error bounds in a rather direct fashion. The only difference is that the factors

$$
\int_{h}^{\rho} r^{-1} d r=\mathcal{O}\left(\ln h^{-1}\right)
$$

must now be replaced by

$$
\int_{h}^{\rho} r^{-p} d r=\mathcal{O}\left(h^{1-p}\right) .
$$

Since the considerations on the uniformity of the convergence remain unaffected, we obtain error bounds of the order $\mathcal{O}\left(h^{\mu-p+2}\right)$ for $f \in C^{\mu+1}(B)$ for both the product quadrature and the quadrature formula of the third kind. Also, the estimate for the formula of the third kind holds uniformly in $B$, whereas the product method does not have this property.

Similar results can be derived when the integral $I_{p}^{*}$ as described in (10) is considered rather than $I_{p}$ as given in (9). 


\subsection{Surface integrals}

Finally, the case of surface integrals with Cauchy singularities, i.e. integrals of the form (1) under the assumption that $B$ is a $d$-dimensional manifold in $\mathbb{R}^{d+1}$, can in principle be reduced to the problem discussed previously by the usual technique of introducing a suitable parametrization, cf. e.g. [14, $\S 8.1 .3 .2]$. The error analysis remains unchanged. However, in practice we encounter the following problem: in view of the parametrization, a Jacobian of the transformation enters the integral. So in order to calculate the value of $Q_{h}[f]$ effectively, we need to integrate the product of the approximating spline and the singularity (as before) and a third factor, namely the Jacobian, analytically. In the general setting, it will often be very difficult to perform this integration in closed form. Nevertheless, this may be a viable path when the transformation is sufficiently simple. In certain cases, computer algebra packages might be invoked to perform the analytic evaluation of the integrals in question.

Acknowledgement: I thank my colleague Guido Walz of the University of Mannheim for very fruitful discussions, and in particular for drawing my attention to reference [2]. 


\section{References}

[1] C. A. Brebbia, J. C. F. Telles, and L. C. Wrobel, Boundary Element Techniques, Springer, Berlin, 1984.

[2] P. G. Ciarlet and P. A. Raviart. General Lagrange and Hermite interpolation in $\mathbb{R}^{n}$ with applications to finite element methods, Arch. Rat. Mech. Anal., 46 (1972), pp. 177-199.

[3] M. G. Cimoroni. Numerical evaluation of a 2-D Cauchy principal value integral based on quasi-interpolating splines, Approx. Theory Appl. (N. S.), 13 (1997), pp. 1-12.

[4] G. Criscuolo and G. Mastroianni. On the uniform convergence of Gaussian quadrature rules for Cauchy principal value integrals, Numer. Math., 54 (1989), pp. 445-461.

[5] C. Dagnino and A. Palamara Orsi. On the evaluation of certain twodimensional singular integrals with Cauchy kernels, Numer. Math., 46 (1985), pp. 121-130.

[6] K. Diethelm. Uniform convergence of optimal order quadrature rules for Cauchy principal value integrals, J. Comput. Appl. Math., 56 (1994), pp. 321-329. 
[7] K. Diethelm. Gaussian quadrature formulae of the third kind for Cauchy principal value integrals: Basic properties and error estimates, J. Comput. Appl. Math., 65 (1995), pp. 97-114.

[8] K. Diethelm. Peano kernels and bounds for the error constants of Gaussian and related quadrature rules for Cauchy principal value integrals, Numer. Math., 73 (1996), pp. 53-63.

[9] K. Diethelm. Error bounds for spline-based quadrature methods for strongly singular integrals, J. Comput. Appl. Math., 89 (1998), pp. 257261.

[10] M. Diligenti and G. Monegato. Finite-part integrals: Their occurrence and computation, Rend. Circ. Mat. Palermo (2) Suppl., 33 (1993), pp. $39-61$.

[11] M. Diligenti and G. Monegato. Integral evaluation in the BEM solution of (hyper)singular integral equations. 2D problems on polygonal domains, J. Comput. Appl. Math., 81 (1997), pp. 29-57.

[12] W. Gautschi, A survey of Gauss-Christoffel quadrature formulae, in E. B. Christoffel: The Influence of his Work on Mathematics and the Physical Sciences, P. L. Butzer and F. Fehér, eds., Basel, 1981, Birkhäuser Verlag, pp. 72-147.

[13] I. G. Graham, W. Hackbusch and S. A. Sauter. Hybrid Galerkin boundary elements: Theory and im- 
plementation, Numer. Math., 86 (2000) pp. 139-172, http://link. springer.de/link/service/journals/00211/bibs/0086001/00860139.h

[14] W. Hackbusch, Integral Equations: Theory and Numerical Treatment, vol. 120 of Internat. Ser. Numer. Math., Birkhäuser, Basel, 1995.

[15] G. Mastroianni and M. R. Occorsio. An algorithm for the numerical evaluation of a Cauchy principal value integral, Ricerche Mat., XXXIII (1984), pp. 3-18.

[16] G. Monegato. Convergence of product formulas for the numerical evaluation of certain two-dimensional Cauchy principal value integrals, Numer. Math., 43 (1984), pp. 161-173.

[17] G. Monegato. The numerical evaluation of a 2-D Cauchy principal value integral arising in boundary integral equation methods, Math. Comp., 62 (1994), pp. $765-777$.

[18] G. Monegato. Numerical evaluation of hypersingular integrals, J. Comput. Appl. Math., 50 (1994), pp. 9-31.

[19] D. Rosen and D. E. Cormack. Singular and near singular integrals in the BEM: A global approach, SIAM J. Appl. Math., 53 (1993), pp. 340-357.

[20] C. Schwab and W. L. Wendland. On numerical cubatures of singular surface integrals in boundary element methods, Numer. Math., 62 (1992), pp. 343-369. 